\title{
"siRNAs and miRNAs": A meeting report on RNA silencing
}

\author{
ZHENGYING HE and ERIK J. SONTHEIMER \\ Department of Biochemistry, Molecular Biology and Cell Biology, Northwestern University, Evanston, Illinois 60208-3500, USA
}

\section{INTRODUCTION}

It has been 11 years since the Ambros laboratory reported the first microRNA (miRNA; Lee et al. 1993), and six years since the Fire and Mello laboratories described the gene silencing effects of double-stranded RNA (dsRNA; Fire et al. 1998). It is now clear that those reports each "lifted a corner of a great veil" (to paraphrase Albert Einstein) that had previously hidden an astonishing range of gene regulatory phenomena, many of which had been hinted at in plants (Matzke and Matzke 2004) and fungi (Pickford et al. 2002). The ongoing efforts to uncover the full biological scope of RNA silencing and to develop it as an experimental and therapeutic tool came into view on April 14-19, 2004, as 540 scientists from around the globe convened in the thin air of Keystone, Colorado, for the 2004 Keystone Symposium entitled "siRNAs and miRNAs." The attendance more than doubled that of a similar Keystone Symposium held two years earlier, attesting to the rapid growth of interest in RNA silencing. Given the ever-expanding breadth of the field, meeting organizers Victor Ambros and Tom Tuschl did an exemplary job of ensuring that there was something for everyone in attendance. The conference was loosely organized around a progression from biological roles to molecular mechanisms, and then on to more applied topics such as large-scale RNA interference (RNAi) screens and dsRNA-based therapeutics. This review will follow the same thematic format, with an emphasis on roles and mechanisms.

\section{BIOLOGY}

The meeting began with keynote addresses from Ron Plasterk (Hubrecht Laboratory) and Andy Fire (Stanford), two pioneers in RNA silencing. Both gave somewhat historical accounts that traced the observations that led them into the field, and both described how their findings prompted them

Reprint requests to: Erik J. Sontheimer, Department of Biochemistry, Molecular Biology and Cell Biology, Northwestern University, 2205 Tech Drive, Evanston, IL 60208-3500, USA; e-mail: erik@northwestern.edu; fax: (847) 491-2467.

Article and publication are at http://www.rnajournal.org/cgi/doi/ 10.1261/rna.7900204. to view RNA silencing as akin to an immune system for the genome. Plasterk described a genetic screen for Caenorhabditis elegans "mutator" strains that fail to silence transposons in the germline. Several mutants identified in this screen also displayed an RNAi defect (Ketting et al. 1999), raising the possibility that one natural role of the RNAi machinery is to protect the genome from invasive nucleic acids. This view has since received direct experimental validation (Sijen and Plasterk 2003). He extended the immune system analogy even further by noting that the roles of RNA-dependent RNA polymerases (RdRPs) in amplifying the dsRNA silencing trigger (in some organisms) can be thought of as a clonal selection step. Fire strengthened this analogy in his address, describing evidence that silencing triggers are amplified only if they encounter their mRNA targets. As one example, he pointed out that only germlineexpressed genes can be silenced beyond the F1 generation in worms, consistent with the idea that the continuous presence of the target may permit ongoing trigger amplification (and therefore ongoing silencing) in subsequent generations.

Plasterk also presented early results from his laboratory's efforts to use miRNAs experimentally in vertebrates. He showed that miRNA injection into zebrafish and Xenopus tropicalis can yield dominant developmental phenotypes without affecting target mRNA levels, implying that the injected miRNAs inhibit gene expression at the level of protein synthesis, as do most natural miRNAs in animals. Mutational analyses indicated that the most important sequence determinants for miRNA specificity and function reside within nt 2-8 at the $5^{\prime}$ end of the mature miRNA. This theme of miRNA target specificity would be echoed by several other speakers later in the meeting.

For the past few years, a primary focus of the miRNA field has been to catalog the complete miRNA inventory in a host of model organisms, using both cloning and bioinformatic approaches (Lai 2003; Bartel 2004). For a few favorite species, the miRNA roster has rapidly expanded and is now beginning to plateau. The identification of these miRNAs has fueled the search for the natural targets of these endogenous regulators, and several talks made clear that the list of validated targets is beginning its own period of rapid expansion. Dave Bartel (Whitehead Institute) be- 
gan with a discussion of miRNA regulation in plants, where most miRNAs are perfectly complementary (or nearly so) to their targets. He reported that the plant miRNA target prediction algorithms have improved to the point that the signal-to-noise ratio is nearing 100:1. Target prediction in animals is also improving, but the hurdles remain significant because, compared to plants, animal miRNAs have a much lower degree of complementarity to their targets. One exception to this apparent rule is the miRNA miR-196, which is nearly perfectly complementary to the mRNA from the mouse homeobox gene HOXB8. Experiments indicate that miR-196 is the first example of a mammalian miRNA that naturally regulates protein production via mRNA degradation rather than translation inhibition (Yekta et al. 2004). Bartel also reported the successful development of miRNA microarrays, which promise to streamline miRNA expression pattern analyses. Preliminary results indicate (not surprisingly) that miRNA tissue specificity is common.

Deborah Marks (Columbia) and Julius Brennecke (from Stephen Cohen's laboratory at the European Molecular Biology Laboratory) each discussed the computational prediction of miRNA targets in Drosophila. Both groups employ algorithms that select targets based on positionweighted complementarity, predicted free energies of miRNA/target duplexes, and target site conservation in related genomes (Enright et al. 2003; Stark et al. 2003). As in other organisms, target prediction in Drosophila is becoming increasingly refined and reliable, and in many cases the predicted targets can be experimentally verified. Marks and colleagues have made their "miRanda" target prediction algorithm available on the web (http://www.microrna.org).

In collaboration with Tom Tuschl's group, Ulrike Gaul's laboratory (Rockefeller) has identified the developmental phenotypes resulting from the loss of miRNA function during Drosophila embryogenesis. By microinjecting a $2^{\prime}-\mathrm{O}-$ methyl RNA oligonucleotide complementary to an individual miRNA, they can specifically inhibit that miRNA's regulatory function. She reported that antisense inhibition of roughly half of the miRNAs known to be expressed in fly embryos yields discernable developmental phenotypes. To cite just a few examples, inhibition of some miRNAs results in massive apoptosis, whereas inhibition of others leads to defects in segmentation, dorsal closure, or both. When combined with bioinformatic target prediction and the enormous knowledge base relevant to Drosophila embryonic development, this approach promises to greatly accelerate miRNA target identification in flies.

Although bioinformatics is a powerful way to predict miRNA targets, classical genetic analyses continue to reveal fascinating examples of miRNA control. Oliver Hobert (Columbia) presented the latest chapter in his laboratory's work on miRNA control of left-right asymmetry in the C. elegans nervous system. His group had previously described how the miRNA lsy-6 down-regulates a transcription factor in the chemosensory neuron ASEL but not in its morphologi- cally bilateral partner neuron ASER (Johnston and Hobert 2003). This control results from the ASEL-specific expression of lsy-6, raising the question of how asymmetric lsy- 6 expression is achieved. They now report that the ASELspecific zinc finger protein DIE-1 drives lsy-6 expression, and that DIE-1 is down-regulated in ASER by yet another miRNA, mir-273. Thus they are working their way up this regulatory heierarchy, and we can look forward to learning how the ASER-restricted expression of mir-273 is specified.

The product of the let-7 locus in C. elegans was the second miRNA ever identified (Reinhart et al. 2000). This miRNA controls certain aspects of developmental timing in part by down-regulating lin-41 expression (Slack et al. 2000). Two other let-7-related genes (mir-48 and mir-241) map to a single 2-kb interval of the $C$. elegans genome, and a genetic screen for suppressors of the lin-4 loss-of-function heterochronic phenotype (Lee et al. 1993) led Ann Rougvie's group (Minnesota) to this locus. She reported that two independent suppressor mutations map to an inverted repeat sequence between mir-48 and mir-241 and result in precocious mir-48 expression. The genetic results suggest that the inverted repeat is a transcriptional control site for mir-48, and that mir-48 cooperates with lin- 4 in controlling the expression of the heterochronic gene lin-28. Victor Ambros (Dartmouth) came to similar conclusions as a result of his laboratory's effort to systematically knock out each miRNA gene in the worm (in collaboration with the Bartel and Horvitz laboratories at MIT). Double and triple knockouts involving mir-48, mir-241, and a third let-7-related locus (mir-84) result in loss of lin-28 control and heterochronic larval development, indicating that let-7 family members function redundantly to time the expression of certain target genes.

In a separate part of his talk, Ambros described the continuing effort to identify novel small RNAs in C. elegans. Computationally predicted miRNAs cannot be considered legitimate until they have been detected experimentally; however, some miRNAs are very weakly expressed and difficult to detect (e.g., lsy-6), complicating their validation. To overcome this problem, his group has employed a new two-color fluorescence-based detection scheme that has improved the sensitivity of miRNA detection by two orders of magnitude. Intriguingly, his laboratory has also identified a class of 22-nt RNAs that are derived from protein-coding sequences and have precise antisense complementarity to the corresponding mRNAs. These endogenous anti-exon siRNAs represent sequences from more than 500 different protein-coding genes, many of which reside on the $\mathrm{X}$ chromosome and are expressed in a germline-enriched manner. He noted that some of these "endo-siRNAs" fail to accumulate in $r r f-3$ mutant worms, and speculated that the decrease in endo-siRNA levels may account for the enhanced RNAi phenotype of $r r f-3$ mutants (Simmer et al. 2002) by reducing competition for potentially limiting RNAi factors. The biological meaning of these endo-siRNAs remains unknown. 
In a separate search for let-7 targets in C. elegans, Frank Slack's laboratory (Yale) found 12 strong candidates. One had 18 potential let-7 binding sites in its $3^{\prime}$-UTR and turned out to be none other than let-60, the worm ortholog of the ras oncogene that plays a critical role in vulval induction (Wang and Sternberg 2001). LET-60 protein expression is limited to the cell that must respond to the inductive signal, and this expression pattern is enforced not by let-7 itself, but by the let-7 family member mir-84. The tumorlike multivulva phenotype that results from LET-60 hyperactivation can be suppressed by mir-84 overexpression, indicating that let-7 family members may function as tumor suppressors. This possibility is underscored by the observation that multiple let-7 binding sites are conserved in the $3^{\prime}$ UTRs of all three human ras genes.

The proposed tumor suppressor function of let-7 family members is not the only potential instance of miRNA function in a process with direct medical relevance. Markus Stoffel's group (Rockefeller) cloned a collection of miRNAs from a pancreatic islet cell line, and identified a previously unknown miRNA called mir-375. This miRNA can readily be detected in primary islet cells as well. Overexpression of mir-375 inhibits glucose-induced insulin secretion, whereas mir-375 inhibition by a complementary $2^{\prime}$-O-methyl RNA has the opposite effect. A series of pharmacological and electrophysiological experiments indicated that mir-375 inhibits insulin secretion at the level of exocytosis, and his group has identified the mRNA from the myotrophin gene as a probable target. Thus it seems possible that miRNA dysfunction could contribute directly to diabetes. Philip Sharp (MIT) and Narry Kim (Seoul National University) both reported the existence of several miRNAs that are specifically expressed in mouse embryonic stem (ES) cells and are repressed during and after differentiation. Although the relevant mRNA targets have not yet been reported, these results suggest that the ES-cell-specific miRNAs may help maintain the "stemness" of this critical population of cells. Tom Tuschl (Rockefeller) presented the results of experiments describing the miRNA population in virus-infected cells (specifically, hepatitis $\mathrm{C}$ virus, yellow fever virus, HIV1, and Epstein-Barr virus; EBV). His group found that virus infection can result in altered levels of endogenous miRNAs, but even more excitingly, they identified five miRNAs that are encoded by the EBV genome itself and are differentially expressed in latent and lytic stages of infection (Pfeffer et al. 2004). These are the first examples of virally encoded miRNAs.

Returning to the plant realm, Jim Carrington (Oregon State) showed that transgenic plants expressing certain viral posttranscriptional gene silencing (PTGS) inhibitors ( $\mathrm{p} 19$, p21, and HC-Pro) increase the expression of some genes that are also up-regulated when miRNA biogenesis is compromised, suggesting that these inhibitors may affect miRNA as well as siRNA function. The p19 protein is known to inhibit PTGS by sequestering siRNA duplexes
(Silhavy et al. 2002), and co-IP and gel-shift experiments indicated that they do the same to miRNAs. The miRNA processing pathway appears to involve a miRNA/miRNA* duplex intermediate, where the miRNA* strand is partially complementary to the mature miRNA, and in most cases does not accumulate to detectable levels. However, in the presence of p19 and p21, both strands accumulate and are bound to the inhibitor (Chapman et al. 2004). In contrast, co-IP experiments indicate that HC-Pro does not associate with either the miRNA or miRNA* strand, suggesting that it acts by a distinct mechanism. The second part of his talk took a more evolutionary turn, as he presented an intriguing model that postulates an inverted duplication mechanism for the evolution of miRNA genes. Transcription through an inverted duplication yields hairpin RNA, and sequence drift could result in imperfect pairing in the premiRNA as well as loss of target similarity in the pre-miRNA arms outside of the mature miRNA sequence itself. This model led him to several predictions, for example, that "young" miRNAs may retain target similarity in flanking regions of the pre-miRNA arms. Preliminary bioinformatic analysis has yielded some interesting examples that are consistent with this model.

Javier Palatnik (from Detlef Weigel's group at the Max Planck Institute for Developmental Biology) also discussed plant miRNA function, and showed that the pleiotropic developmental defects of the jaw-D insertion mutant result from overexpression of a miRNA (miR-JAW) that is encoded by a nearby locus (Palatnik et al. 2003). Microarray analysis revealed that five TCP transcription factor genes are down-regulated in jaw-D plants, and that all five share a 20-nt sequence that is complementary to miR-JAW. Several lines of evidence confirm that TCP transcription factor levels are directly modulated by miR-JAW-directed mRNA degradation.

Although miRNAs dominated the discussion of RNA silencing's biological functions, some other important themes emerged as well. Alexander Hüttenhofer (Innsbruck) described his "experimental RNomics" approach for defining the small nonmessenger RNA (snmRNA) population in many different model organisms and organelles (Hüttenhofer et al. 2004). His laboratory has uncovered a host of snmRNAs, many of which are tissue or organelle specific, and has also noted some clues to their functions. As one example, his group has found a set of small brain-specific RNAs from humans that map to a chromosome region implicated in the neurodegenerative disease Prader-Willi Syndrome (Cavaille et al. 2000). Interestingly, one is complementary to a region of a serotonin receptor mRNA that is posttranscriptionally edited and alternatively spliced. Although his group's efforts are focused on RNAs outside of the 20-25-nt size range, and although he did not address RNA silencing as such, his talk served as a healthy reminder that many other regulatory RNAs exist in addition to miRNAs and siRNAs. 
Andres Verdel (from Danesh Moazed's laboratory at Harvard) presented work done in collaboration with Shiv Grewal's laboratory (now at the National Cancer Institute) that has defined a new effector complex that modifies chromatin in Schizosaccharomyces pombe. Heterochromatin formation in this organism requires the RNAi pathway (Hall et al. 2002; Volpe et al. 2002; Schramke and Allshire 2003), which now appears to work through an assembly called the

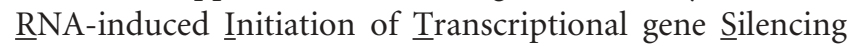
(RITS) complex (Verdel et al. 2004). This complex associates directly with heterochromatin and includes Agol (the lone PIWI/PAZ protein in fission yeast), the heterochromatin protein Chp1, the novel protein Tas3, and 21-23-nt RNAs that are derived from heterochromatic regions such as centromeres. The RNAs appear to target the RITS complex to the proper chromosomal locations, though it is unclear whether they recognize the DNA directly by base pairing.

Other fates besides Dicer-mediated processing can befall dsRNA molecules. One such fate is RNA editing (in particular, adenosine deamination by ADAR enzymes), and Brenda Bass (Utah) presented her laboratory's work on functional intersections between the RNAi and editing pathways. She described some extensively base-paired and edited C. elegans transcripts that act as competitive inhibitors of Dicer in vitro. Her group found that in some cases the extent of editing of these RNAs affects their export into the cytoplasm (where most Dicer resides). She suggested that cells may use RNA editing to modulate export, thus providing a mechanism for regulated Dicer inhibition, as well as regulated translation of the editing substrates. The known susceptibility of ADARs to substrate inhibition provides a plausible way for cells to modulate ADAR activity. This sort of functional interplay might account for the known genetic interactions between mutations that affect the two pathways (Tonkin and Bass 2003).

C. elegans uses additional strategies to modulate RNAi activity in different tissues. Neural mRNAs are often refractory to RNAi, and Gary Ruvkun (Harvard) described a genetic screen for mutants with enhanced RNAi activity in neurons. The screen led his group to identify a novel gene called eri-1 that encodes a nuclease that specifically cleaves the 3' overhangs from siRNAs (Kennedy et al. 2004), thereby blocking efficient siRNA incorporation into effector complexes. Loss-of-function eri-1 mutants exhibit a temperature-sensitive sterile phenotype, as do mutants in another known RNAi suppressor gene, $r r f-3$ (Simmer et al. 2002). This observation, and the fact that eri-1 and rrf-3 mutations do not reciprocally enhance each other's phenotype, suggest that ERI-1 and RRF-3 may function in the same pathway.

\section{MECHANISM}

Uncovering the mechanisms of the numerous RNA silencing pathways will be necessary both to understand their biological roles and to co-opt them for experimental and therapeutic purposes. Accordingly, many of the talks were devoted to explaining the biochemical mechanisms of RNA silencing, and completing the roster of genes and proteins involved. Craig Mello (University of Massachusetts) reported the latest results from his laboratory's screen for RNAi-defective ( $r d e$ ) mutants in C. elegans. They have now cloned the $r d e-3$ locus, and found that it encodes a member of the polymerase $\beta$ family that includes poly $(\mathrm{A})$ polymerases and $2^{\prime}-5^{\prime}$ oligo(A) synthetases. The mechanistic basis for the RNAi defect in $r d e-3$ mutants is not yet clear. He also continued his description of a set of proteins (DCR-1, RDE1, RDE-4, and DRH-1/2) involved in RNAi initiation in worms (Tabara et al. 2002). He reported new data showing that these proteins all reside within a single complex that exhibits dsRNA processing activity, making it very likely that this complex is directly analogous to one in Drosophila that initiates dsRNA processing and RNA-induced silencing complex (RISC) assembly (Liu et al. 2003; Pham et al. 2004; Tomari et al. 2004). RDE-1 is one of $27 \mathrm{PIWI} / \mathrm{PAZ}$ proteins in the worm genome, and his group has now generated null alleles of all but six of them. Certain PIWI/PAZ null mutants display very interesting and novel phenotypes, including chromosome segregation defects.

A genetic screen for RNAi-defective mutants is now being conducted in Drosophila as well, as reported in a talk by Young Sik Lee (from Rich Carthew's laboratory at Northwestern). Although this approach promises to identify novel RNAi factors in flies, the first fruits of the screen have come from analyses of mutants that lack either of the known RNAi factors Dicer-1 or Dicer-2 (Lee et al. 2004). His data showed that only Dicer-1 is essential for miRNA processing, whereas Dicer-2 plays a dominant role in the siRNA pathway in vivo and in vitro. He described proteomic experiments that identified at least 20 proteins that are reproducibly up-regulated in dicer-1 null mutant embryos, suggesting that they are targets of miRNA-mediated inhibition. As for the RNAi pathway, he found that the defect exhibited by dicer-2 null mutants persists even when RNAi is triggered by "pre-diced" siRNAs, implying that Dicer-2's function in RNAi is not limited to dsRNA processing. Instead, biochemical experiments with dicer-2 null mutant embryo lysates revealed that siRNA/protein complexes fail to form in the absence of Dicer-2, suggesting a critical role for Dicer-2 in RISC assembly. One of us (E.J. Sontheimer) picked up on this theme in a short talk that described a RISC assembly pathway involving at least three Dicer-2-dependent siRNA/ protein complexes (Pham et al. 2004). Exogenous siRNAs initially associate with Dicer-2 in a complex that cofractionates with dsRNA processing activity, and the assembly pathway culminates in a very large $(\sim 80 \mathrm{~S})$ form of RISC that, surprisingly, contains Dicer-2 itself. These observations indicate that Dicer-2 does not "hand off" newly processed siRNAs to a distinct complex, but rather assembles into effector complexes along with the siRNA. 
Qinghua Liu (Texas-Southwestern) described work done in Xiaodong Wang's laboratory on the Drosophila protein R2D2, which he identified as a Dicer-2-associated RNAi factor (Liu et al. 2003). Although Dicer-2 alone can process dsRNAs into siRNAs, he showed that R2D2 is required for siRNA incorporation into RISC, and that neither protein is stably expressed in the absence of the other. Recombinant Dicer-2/R2D2 heterodimer readily binds siRNAs, unlike Dicer-2 alone. He described microinjection assays in $r 2 d 2$ null mutant embryos that confirm R2D2's role in RNAi in vivo. Unlike the dicer-2 mutants, $r 2 d 2$ mutant animals exhibit developmental phenotypes, suggesting at least some functional distinctions between the two proteins.

Witek Filipowicz (Friedrich Miescher Institute) presented fascinating new results from his ongoing analysis of the human Dicer (hDicer) protein. His group has characterized recombinant proteins with mutations in predicted active-site residues (based on the crystal structure of Aquifex aeolicus RNase III; Blaszczyk et al. 2001), and he reported results that are not consistent with the previously described enzyme/dsRNA structural model. Instead he discussed a compelling scenario in which an hDicer monomer associates with a dsRNA terminus (probably via PAZ domain binding), and then each of the two RNase III domains makes a single endonucleolytic cut 21-23 nt from the bound end. The model was particularly interesting in light of new results presented by Phil Zamore (University of Massachusetts), whose group used site-specific photocrosslinking with chemically synthesized siRNAs to examine protein/siRNA interactions. His laboratory (Schwarz et al. 2003) and others (Khvorova et al. 2003) had previously reported that siRNA loading into RISC is asymmetric if the duplex termini have distinct thermal stabilities (specifically, the strand whose $5^{\prime}$ terminus is at the less stable end is preferentially incorporated). By placing the photoactivatable group at either end of an asymmetric siRNA, they found that Dicer-2 interactions are also asymmetric, with cross-linking observed at the $5^{\prime}$ end of the strand that is destined to enter RISC. The other end of the RISC-bound strand interacts with R2D2 and an unidentified 120-kDa protein. He proposed that the Dicer-2/ R2D2-containing complex that initiates RISC assembly (Liu et al. 2003; Pham et al. 2004; Tomari et al. 2004) is required for siRNA unwinding and interprets siRNA asymmetry.

Dicer's roles in RNAi figured heavily in each of the six talks just described, and a new and recurring theme was that Dicer/siRNA interactions are critical for RISC assembly. Because Dicer is also required to generate siRNA (Bernstein et al. 2001), an appealing model is that Dicer remains bound to the newly generated siRNA after the dsRNA processing step, and then ferries it directly into the RISC complex. However, if Dicer binds the dsRNA substrate end asymmetrically, then after dsRNA cleavage to generate siRNA, it will be bound in the "correct" orientation (such that it directs unwinding from the least stable end) only half the time. Therefore, if the relative-end-stability model of siRNA strand selection is correct, then Dicer must release those siRNAs that are bound in the "incorrect" orientation, so that they can then rebind in the "correct" orientation. A critical issue is whether the relative-end-stability model (which was based on studies with synthetic siRNAs that do not undergo dsRNA processing) also holds for reactions with longer triggers. At least one published experiment suggests that strand asymmetry with longer dsRNA triggers may be dictated primarily by the direction of Dicer processing (Elbashir et al. 2001). These issues came up repeatedly during the question-and-answer sessions, and will clearly be the subject of future work.

Filipowicz's Dicer/dsRNA model included PAZ domain binding to the dsRNA terminus in part because the PAZ domains from two different Drosophila PIWI/PAZ proteins were recently shown to adopt a nucleic-acid-binding fold that exhibits end-binding activity (Lingel et al. 2003; Song et al. 2003; Yan et al. 2003). Building on this earlier work, Keqiong Ye (from Dinshaw Patel's laboratory at Memorial Sloan-Kettering) described the crystal structure of a PAZ domain bound to a short RNA duplex bearing 2-nt 3' overhangs (Ma et al. 2004). The overhangs fit into a binding pocket and contact residues that are nearly invariant in PAZ domains, suggesting that the interactions are functionally meaningful. He also described direct binding studies that confirm the contribution of the $3^{\prime}$ overhangs to PAZ domain binding affinity.

Most species produce numerous PIWI/PAZ proteins, and there is already considerable evidence for functional distinctions between individual family members within each species. Greg Hannon (Cold Spring Harbor) discussed gene knockout results in mice that show that distinct phenotypes are associated with loss of individual PIWI/PAZ paralogs. One $(\mathrm{Ago} 3 \Delta)$ is viable, another (Miwi2 $\Delta$ ) is viable but male-sterile due to spermatogenesis defects, and a third $($ Ago $2 \Delta)$ dies with multiple developmental abnormalities. Pull-down experiments using tagged constructs in human cells suggest that different PIWI/PAZ proteins reside in distinct complexes, because they do not all display the same biochemical activities.

In addition to the viral miRNAs noted above, Tom Tuschl's talk included recent findings about the target cleavage activity associated with minimal human RISC (Martinez et al. 2002). Because the affinity-purified complex is free of contaminating exonucleases, they can assay cleavage of small, uncapped, synthetic RNAs, enabling them to define minimal substrates and introduce precise chemical modifications. They found that human RISC is a true endonuclease that leaves $5^{\prime}$-phosphate and 3 '-hydroxyl termini (Martinez and Tuschl 2004), consistent with recent results obtained with Drosophila RISC (Schwarz et al. 2004). This finding places chemical constraints on the cleavage mechanism, which could be very valuable in evaluating future 
candidates in the search for "Slicer," the RISC-associated cleavage enzyme.

Unlike RNAi, miRNA-mediated inhibition of protein synthesis has not yet been reported in vitro, and as a result fewer mechanistic advances were reported for the miRNA pathway. Nonetheless, some important information emerged about miRNA function and biogenesis. In a second part of his talk, Witek Filipowicz showed that miRNAindependent tethering of certain human PIWI/PAZ proteins to the $3^{\prime}$ UTR of a reporter mRNA suffices for target inhibition in cultured cells in a manner indistinguishable from that of miRNAs. Importantly, this result reveals that the miRNAs themselves serve only as guides, and that the annealed miRNA/target structure does not play a direct role in silencing. Phil Sharp described experiments with cultured mammalian cells expressing two reporter genes, only one of which is subject to miRNA down-regulation. Sucrose gradient analyses revealed that mRNAs from both reporters were loaded onto polysomes (consistent with previous results from C. elegans; Olsen and Ambros 1999), and both shifted into the same lighter fractions upon treatment with translation inhibitors. The fact that the two mRNAs behave identically, even when only one is inhibited by miRNAs, led him to speculate that miRNAs might not affect translation directly, but instead might somehow direct the cotranslational degradation of the nascent polypeptide. His group was unable to detect degradation intermediates, and they also observed that miRNA silencing persisted in the presence of proteasome inhibitors. Therefore, if his suggested mechanism is correct, then miRNA-directed degradation is likely to be very efficient and proteasome independent.

Narry Kim presented important new results on miRNA expression, and has now obtained considerable evidence that miRNA precursors are generated by RNA polymerase II. By using eIF4E affinity chromatography, her group showed that nonintronic miRNA precursors ("primiRNAs"; Lee et al. 2003) have a 7-methylguanosine cap, which is a structure specific to RNA polymerase II transcripts. Her laboratory has now mapped the 5 ' terminus of at least one mammalian pri-miRNA, and has confirmed that the region upstream of that site is a promoter that can drive expression of a heterologous protein-encoding reporter gene. While examining one of his laboratory's new ES-cell-specific miRNAs (see above), Phil Sharp's group also obtained data consistent with RNA polymerase II transcription and pri-miRNA capping, and has mapped the 5' terminus and analyzed the corresponding promoter. Thus, the effort to understand miRNA transcriptional control has now commenced.

\section{EXPERIMENTAL AND THERAPEUTIC APPLICATION}

One of the most important consequences of the RNA silencing revolution is the ability to use the pathways to de- termine gene function and (hopefully someday) treat disease. Of course, mechanistic understanding improves the prospects for application, and the lessons learned during technique development can provide important clues about mechanism, so the distinctions are not always clear-cut. We saw several examples of this during the symposium.

RNAi in mammalian cells can be achieved with synthetic siRNA, but this has often been a hit-or-miss process: Some siRNAs work beautifully, and others do not work at all (Mittal 2004). Anastasia Khvorova (Dharmacon) described a large-scale effort to identify siRNA features that maximize knockdown efficiency (Reynolds et al. 2004), which has led to an algorithm that incorporates 66 such parameters and has greatly improved the reliability of siRNA design. Along the way they have identified some siRNAs that she described as "hyperfunctional" in cultured cells, with effective concentrations in the low picomolar range and silencing longevities of $10 \mathrm{~d}$ or more. She also mentioned a proprietary chemical modification that blocks RISC incorporation by the siRNA sense strand but not the antisense strand, reducing the likelihood of sense-strand-directed off-target effects (see below). Iwao Nozawa (GenoFunction) presented a system for generating plasmid libraries encoding short hairpin RNAs (shRNAs), which in principle can be subjected to an iterative screening procedure to identify the most active silencing triggers in the library. This has the potential to allow the prediction/selection process to be bypassed.

Greg Hannon and coworkers were among the first to introduce shRNA vectors (Paddison et al. 2002). More recently they have developed an shRNA-based system to facilitate genome-wide functional screens in mammalian cells. Though the ink is barely dry on the paper reporting their first such system (Paddison et al. 2004), he described some features of a second-generation library that is under development. The new system is designed to capitalize on improved shRNA design rules, incorporate features that promote efficient shRNA processing, and permit tissue-specific or inducible expression. Preliminary results indicate that these changes can improve the penetrance and hit rate when compared to screens conducted with the first-generation system. René Bernards and colleagues (Netherlands Cancer Institute) recently reported comparable large-scale approaches to RNAi screens in human cells (Berns et al. 2004), and his talk discussed their continuing screens for anticancer drug targets. Their latest adaptation is a screen for RNAi triggers that overcome the effects of growth inhibitory signals, and he described some promising candidates from these screens. Mark Edbrooke (GlaxoSmithKline) presented additional examples of high-throughput RNAi approaches in drug target identification and validation.

Some cautionary reports have appeared describing partial silencing of transcripts other than the intended target, based on microarray assays (Jackson et al. 2003; Persengiev et al. 
2004; Scacheri et al. 2004). This has led to concern over the potential frequency of false positives in RNAi-based functional screens. Peter Linsley (Rosetta Inpharmatics) discussed his group's work on expression profiling of siRNAtreated cells, and reported that new siRNA/shRNA design rules can improve the situation in some cases, but that off-target effects remain a significant issue. During the question period that followed the talk, some noted that proof-of-principle shRNA screens for components of wellcharacterized pathways did not suffer from a high rate of false positives (Berns et al. 2004; Paddison et al. 2004), and suggested that microarray expression profiling may provide a far more sensitive readout than a typical functional screen. The consensus view at the end seemed to be that the potential for off-target effects should certainly be borne in mind, but that it should not stop others from employing RNAi as an experimental or pharmacological tool as long as they are appropriately cautious in their interpretations.

Genome-wide RNAi screens are obviously very useful in model organisms as well as cultured mammalian cells, and Christophe Echeverri (Cenix Bioscience) presented his group's large-scale RNAi screens for a range of scorable phenotypes in C. elegans. He discussed the advantages of multiparameter screens (i.e., those in which multiple phenotypic readouts are employed), and as an example, he presented a video-microscopy-based screen for genes required for cell division in one- to four-cell embryos. They have succeeded in identifying more than 600 genes that, when knocked down, can be placed into one or more of 47 distinct defect categories. More than 60 genes are known to be essential for early embryonic development in the worm, and the screen picked up $\sim 95 \%$ of them, indicating a low rate of false negatives.

David Baulcombe's group (Sainsbury Laboratory) has been developing tools for functional genomics in plants, and he discussed their establishment of potato virus $\mathrm{X}$ (PVX) as an effective vector for high-throughput virus-induced gene silencing (Lu et al. 2003). Conveniently, cDNA inserts can trigger silencing of corresponding plant target genes, and it is not even necessary to clone the inserts as inverted repeats. PVX-based libraries have now been used in screens that score for a range of phenotypes, demonstrating the potential of the approach. He also presented a genetic screen for mutants that show enhanced silencing in plants, and interestingly, several such mutants map to genes implicated in mRNA processing. He described a model in which defects in splicing or polyadenylation could increase the population of aberrant transcripts that then enter the silencing pathway, thereby potentiating the response. Michael Metzlaff (Bayer Bioscience) reported that his group has developed plant satellite viruses as silencing vectors (Gossele et al. 2002), and noted that the satellite viruses can be present in 50- to 100 -fold excess over the accompanying autonomous viruses. Satellite virus coinfection can easily be established with in vitro transcripts, and his group has used this system to induce the systemic silencing of multiple genes with a broad range of expression levels.

Of course, viruses are currently envisioned as targets as well as vectors for RNA silencing. Mark Kay's laboratory (Stanford) previously achieved knockdown of hepatitis B virus gene expression in adult mice by hydrodynamic siRNA injection (McCaffrey et al. 2002). Despite their success, they recognized the need for delivery approaches that are feasible for human therapies. He now reports that a specific pseudotype of adeno-associated virus is taken up by nearly $100 \%$ of hepatocytes in vivo, and that nearly all infected hepatocytes express shRNA constructs inserted into the viral genome. Despite some potential problems with viral toxicity, target transcript levels were reduced by more than $98 \%$, suggesting that later generations of these vectors could be useful in the treatment of liver infections. Muthiah Manoharan (Alnylam Pharmaceuticals) and David Morrissey (Sirna Therapeutics) each presented their respective group's progress in developing synthetic siRNA drugs. Both groups are also attempting to direct siRNA to the liver, in part to meet the unmet clinical need presented by hepatitis $\mathrm{C}$ virus infection. Both are developing proprietary chemical modifications that improve serum stability and tissue uptake, and Manoharan discussed a small molecule natural product that is taken up by hepatocytes and that can be chemically conjugated to the $3^{\prime}$ terminus of an siRNA passenger strand. Despite their reported advances, both were very forthright about the significant hurdles that remain in developing siRNA-based therapeutics, especially with regard to delivery. Sailen Barik (South Alabama) presented his laboratory's work using RNAi to dissect host/pathogen interactions for viruses and for protozoan parasites, with a particular emphasis on membrane fusion in respiratory syncytial virus (RSV). He characterized viral and host proteins that modulate the actin cytoskeleton in ways that help drive membrane fusion, and identified antiviral siRNAs that suppress this important step in viral pathogenesis.

To no one's surprise, all stages of the meeting reaffirmed the sense that the excitement over RNA silencing will continue for the foreseeable future. Large numbers of miRNAbased regulatory circuits remain undiscovered, as does the very mechanism of miRNA silencing in animals. Some basic biochemical features of the RNAi pathway are coming into focus, but most of the fundamental mechanistic questions persist. Our understanding of chromatin-based transcriptional silencing is in an even more primitive state. These and other aspects of this young field continue to provide fertile scientific ground, and the resulting insights promise to enhance the effort to apply RNA silencing as a tool for research, agriculture, and medicine.

\section{REFERENCES}

Bartel, D.P. 2004. MicroRNAs: Genomics, biogenesis, mechanism, and function. Cell 116: 281-297. 
Berns, K., Hijmans, E.M., Mullenders, J., Brummelkamp, T.R., Velds, A., Heimerikx, M., Kerkhoven, R.M., Madiredjo, M., Nijkamp, W., Weigelt, B., et al. 2004. A large-scale RNAi screen in human cells identifies new components of the p53 pathway. Nature 428: 431437.

Bernstein, E., Caudy, A.A., Hammond, S.M., and Hannon, G.J. 2001. Role for a bidentate ribonuclease in the initiation step of RNA interference. Nature 409: 363-366.

Blaszczyk, J., Tropea, J.E., Bubunenko, M., Routzahn, K.M., Waugh, D.S., Court, D.L., and Ji, X. 2001. Crystallographic and modeling studies of RNase III suggest a mechanism for double-stranded RNA cleavage. Structure 9: 1225-1236.

Cavaille, J., Buiting, K., Kiefmann, M., Lalande, M., Brannan, C.I., Horsthemke, B., Bachellerie, J.P., Brosius, J., and Hüttenhofer, A. 2000. Identification of brain-specific and imprinted small nucleolar RNA genes exhibiting an unusual genomic organization. Proc. Natl. Acad. Sci. 97: 14311-14316.

Chapman, E.J., Prokhnevsky, A.I., Gopinath, K., Dolja, V.V., and Carrington, J.C. 2004. Viral RNA silencing suppressors inhibit the microRNA pathway at an intermediate step. Genes \& Dev. 18: 1179-1186.

Elbashir, S.M., Lendeckel, W., and Tuschl, T. 2001. RNA interference is mediated by 21- and 22-nucleotide RNAs. Genes \& Dev. 15: 188200.

Enright, A.J., John, B., Gaul, U., Tuschl, T., Sander, C., and Marks, D.S. 2003. MicroRNA targets in Drosophila. Genome Biol. 5: R1.1R1.14.

Fire, A., Xu, S., Montgomery, M.K., Kostas, S.A., Driver, S.E., and Mello, C.C. 1998. Potent and specific genetic interference by double-stranded RNA in Caenorhabditis elegans. Nature 391: 806811.

Gossele, V., Fache, I., Meulewaeter, F., Cornelissen, M., and Metzlaff, M. 2002. SVISS-A novel transient gene silencing system for gene function discovery and validation in tobacco plants. Plant J. 32: 859-866.

Hall, I.M., Shankaranarayana, G.D., Noma, K.I., Ayoub, N., Cohen, A., and Grewal, S.I. 2002. Establishment and maintenance of a heterochromatin domain. Science 297: 2232-2237.

Hüttenhofer, A., Cavaille, J., and Bachellerie, J.P. 2004. Experimental RNomics: A global approach to identifying small nuclear RNAs and their targets in different model organisms. Methods Mol. Biol. 265: 409-428.

Jackson, A.L., Bartz, S.R., Schelter, J., Kobayashi, S.V., Burchard, J., Mao, M., Li, B., Cavet, G., and Linsley, P.S. 2003. Expression profiling reveals off-target gene regulation by RNAi. Nat. Biotechnol. 21: 635-637.

Johnston, R.J. and Hobert, O. 2003. A microRNA controlling left/right neuronal asymmetry in Caenorhabditis elegans. Nature 426: 845849.

Kennedy, S., Wang, D., and Ruvkun, G. 2004. A conserved siRNAdegrading RNase negatively regulates RNA interference in $\mathrm{C}$. elegans. Nature 427: 645-649.

Ketting, R.F., Haverkamp, T.H., van Luenen, H.G., and Plasterk, R.H. 1999. Mut-7 of C. elegans, required for transposon silencing and RNA interference, is a homolog of Werner syndrome helicase and RNaseD. Cell 99: 133-141.

Khvorova, A., Reynolds, A., and Jayasena, S.D. 2003. Functional siRNAs and miRNAs exhibit strand bias. Cell 115: 209-216.

Lai, E.C. 2003. MicroRNAs: Runts of the genome assert themselves. Curr. Biol. 13: R925-R936.

Lee, R.C., Feinbaum, R.L., and Ambros, V. 1993. The C. elegans heterochronic gene lin-4 encodes small RNAs with antisense complementarity to lin-14. Cell 75: 843-854.

Lee, Y., Ahn, C., Han, J., Choi, H., Kim, J., Yim, J., Lee, J., Provost, P., Radmark, O., Kim, S., et al. 2003. The nuclear RNase III Drosha initiates microRNA processing. Nature 425: 415-419.

Lee, Y.S., Nakahara, K., Pham, J.W., Kim, K., He, Z., Sontheimer, E.J., and Carthew, R.W. 2004. Distinct roles for Drosophila Dicer-1 and Dicer-2 in the siRNA/miRNA silencing pathways. Cell 117: 69-81.
Lingel, A., Simon, B., Izaurralde, E., and Sattler, M. 2003. Structure and nucleic-acid binding of the Drosophila Argonaute 2 PAZ domain. Nature 426: 465-469.

Liu, Q., Rand, T.A., Kalidas, S., Du, F., Kim, H.E., Smith, D.P., and Wang, X. 2003. R2D2, a bridge between the initiation and effector steps of the Drosophila RNAi pathway. Science 301: 1921-1925.

Lu, R., Malcuit, I., Moffett, P., Ruiz, M.T., Peart, J., Wu, A.J., Rathjen, J.P., Bendahmane, A., Day, L., and Baulcombe, D.C. 2003. High throughput virus-induced gene silencing implicates heat shock protein 90 in plant disease resistance. EMBO J. 22: 5690-5699.

Ma, J.-B., Ye, K., and Patel, D.J. 2004. Structural basis for overhangspecific small interfering RNA recognition by the PAZ domain. Nature 429: 318-322.

Martinez, J. and Tuschl, T. 2004. RISC is a $5^{\prime}$ phosphomonoesterproducing RNA endonuclease. Genes \& Dev. 18: 975-980.

Martinez, J., Patkaniowska, A., Urlaub, H., Luhrmann, R., and Tuschl, T. 2002. Single-stranded antisense siRNAs guide target RNA cleavage in RNAi. Cell 110: 563-574.

Matzke, M.A. and Matzke, A.J.M. 2004. Planting the seeds of a new paradigm. PLoS Biol. 2: 582-586.

McCaffrey, A.P., Meuse, L., Pham, T.T., Conklin, D.S., Hannon, G.J., and Kay, M.A. 2002. RNA interference in adult mice. Nature 418: $38-39$.

Mittal, V. 2004. Improving the efficiency of RNA interference in mammals. Nat. Rev. Genet. 5: 355-365.

Olsen, P.H. and Ambros, V. 1999. The lin-4 regulatory RNA controls developmental timing in Caenorhabditis elegans by blocking LIN14 protein synthesis after the initiation of translation. Dev. Biol. 216: 671-680.

Paddison, P.J., Caudy, A.A., Bernstein, E., Hannon, G.J., and Conklin, D.S. 2002. Short hairpin RNAs (shRNAs) induce sequence-specific silencing in mammalian cells. Genes \& Dev. 16: 948-958.

Paddison, P.J., Silva, J.M., Conklin, D.S., Schlabach, M., Li, M., Aruleba, S., Balija, V., O’Shaughnessy, A., Gnoj, L., Scobie, K., et al. 2004. A resource for large-scale RNA-interference-based screens in mammals. Nature 428: 427-431.

Palatnik, J.F., Allen, E., Wu, X., Schommer, C., Schwab, R., Carrington, J.C., and Weigel, D. 2003. Control of leaf morphogenesis by microRNAs. Nature 425: 257-263.

Persengiev, S.P., Zhu, X., and Green, M.R. 2004. Nonspecific, concentration-dependent stimulation and repression of mammalian gene expression by small interfering RNAs (siRNAs). RNA 10: 12-18.

Pfeffer, S., Zavolan, M., Grasser, F.A., Chien, M., Russo, J.J., Ju, J., John, B., Enright, A.J., Marks, D., Sander, C., et al. 2004. Identification of virus-encoded microRNAs. Science 304: 734-736.

Pham, J.W., Pellino, J.L., Lee, Y.S., Carthew, R.W., and Sontheimer, E.J. 2004. A Dicer-2-dependent 80 S complex cleaves targeted mRNAs during RNAi in Drosophila. Cell 117: 83-94.

Pickford, A.S., Catalanotto, C., Cogoni, C., and Macino, G. 2002. Quelling in Neurospora crassa. Adv. Genet. 46: 277-303.

Reinhart, B.J., Slack, F.J., Basson, M., Pasquinelli, A.E., Bettinger, J.C. Rougvie, A.E., Horvitz, H.R., and Ruvkun, G. 2000. The 21-nt let-7 RNA regulates developmental timing in C. elegans. Nature 403: 901-906.

Reynolds, A., Leake, D., Boese, Q., Scaringe, S., Marshall, W.S., and Khvorova, A. 2004. Rational siRNA design for RNA interference. Nat. Biotechnol. 22: 326-330.

Scacheri, P.C., Rozenblatt-Rosen, O., Caplen, N.J., Wolfsberg, T.G., Umayam, L., Lee, J.C., Hughes, C.M., Shanmugam, K.S., Bhattacharjee, A., Meyerson, M., et al. 2004. Short interfering RNAs can induce unexpected and divergent changes in the levels of untargeted proteins in mammalian cells. Proc. Natl. Acad. Sci. 101: 1892-1897.

Schramke, V. and Allshire, R. 2003. Hairpin RNAs and retrotransposon LTRs effect RNAi and chromatin-based gene silencing. Science 301: 1069-1074.

Schwarz, D.S., Hutvagner, G., Du, T., Xu, Z., Aronin, N., Zamore, P.D. 2003. Asymmetry in the assembly of the RNAi enzyme complex. Cell 115: 199-208. 
Schwarz, D.S., Tomari, Y., and Zamore, P.D. 2004. The RNA-induced silencing complex is a $\mathrm{Mg}(2+)$-dependent endonuclease. Curr. Biol. 14: 787-791.

Sijen, T. and Plasterk, R.H. 2003. Transposon silencing in the Caenorhabditis elegans germ line by natural RNAi. Nature 426: 310-314.

Silhavy, D., Molnar, A., Lucioli, A., Szittya, G., Hornyik, C., Tavazza, M., and Burgyan, J. 2002. A viral protein suppresses RNA silencing and binds silencing-generated, 21- to 25-nucleotide doublestranded RNAs. EMBO J. 21: 3070-3080.

Simmer, F., Tijsterman, M., Parrish, S., Koushika, S., Nonet, M., Fire, A., Ahringer, J., and Plasterk, R. 2002. Loss of the putative RNAdirected RNA polymerase RRF-3 makes C. elegans hypersensitive to RNAi. Curr. Biol. 12: 1317.

Slack, F.J., Basson, M., Liu, Z., Ambros, V., Horvitz, H.R., and Ruvkun, G. 2000. The lin-41 RBCC gene acts in the C. elegans heterochronic pathway between the let-7 regulatory RNA and the LIN29 transcription factor. Mol. Cell 5: 659-669.

Song, J.J., Liu, J., Tolia, N.H., Schneiderman, J., Smith, S.K., Martienssen, R.A., Hannon, G.J., and Joshua-Tor, L. 2003. The crystal structure of the Argonaute2 PAZ domain reveals an RNA binding motif in RNAi effector complexes. Nat. Struct. Biol. 10: 1026-1032.

Stark, A., Brennecke, J., Russell, R.B., and Cohen, S.M. 2003. Identification of Drosophila microRNA targets. PLoS Biol. 1: E60-E72.
Tabara, H., Yigit, E., Siomi, H., and Mello, C.C. 2002. The dsRNA binding protein RDE-4 interacts with RDE-1, DCR-1, and a DExH-box helicase to direct RNAi in C. elegans. Cell 109: 861-871.

Tomari, Y., Du, T., Haley, B., Schwarz, D.S., Bennett, R., Cook, H.A., Koppetsch, B.S., Theurkauf, W.E., and Zamore, P.D. 2004. RISC assembly defects in the Drosophila RNAi mutant armitage. Cell 116: $831-841$

Tonkin, L.A. and Bass, B.L. 2003. Mutations in RNAi rescue aberrant chemotaxis of ADAR mutants. Science 302: 1725.

Verdel, A., Jia, S., Gerber, S., Sugiyama, T., Gygi, S., Grewal, S.I.S., and Moazed, D. 2004. RNAi-mediated targeting of heterochromatin by the RITS complex. Science 303: 672-676.

Volpe, T.A., Kidner, C., Hall, I.M., Teng, G., Grewal, S.I., and Martienssen, R.A. 2002. Regulation of heterochromatic silencing and histone H3 lysine-9 methylation by RNAi. Science 297: 18331837.

Wang, M. and Sternberg, P.W. 2001. Pattern formation during C. elegans vulval induction. Curr. Top. Dev. Biol. 51: 189-220.

Yan, K.S., Yan, S., Farooq, A., Han, A., Zeng, L., and Zhou, M.M. 2003. Structure and conserved RNA binding of the PAZ domain. Nature 426: 468-474.

Yekta, S., Shih, I.H., and Bartel, D.P. 2004. MicroRNA-directed cleavage of HOXB8 mRNA. Science 304: 594-596. 

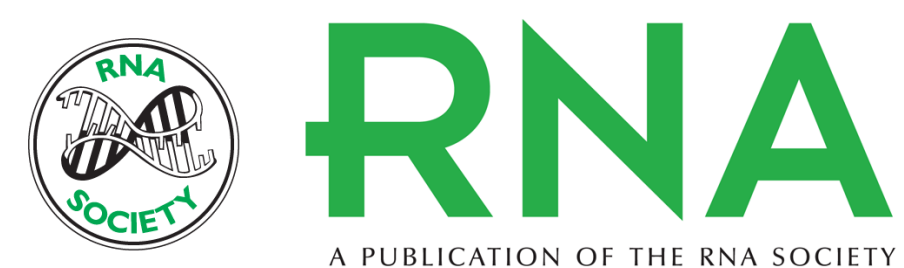

A PUBLICATION OF THE RNA SOCIETY

\section{"siRNAs and miRNAs": A meeting report on RNA silencing}

\section{ZHENGYING HE and ERIK J. SONTHEIMER}

RNA 2004 10: 1165-1173

\section{References This article cites 57 articles, 17 of which can be accessed free at:} http://rnajournal.cshlp.org/content/10/8/1165.full.html\#ref-list-1

\section{License}
Email Alerting Receive free email alerts when new articles cite this article - sign up in the box at the Service top right corner of the article or click here.

To subscribe to $R N A$ go to:

http://rnajournal.cshlp.org/subscriptions 